\title{
Virtual Reality Surgical Training in Ear, Nose and Throat Surgery
}

\author{
Patorn Piromchai 1 , \\ ${ }^{1}$ Department of Otolaryngology, Royal Victorian Eye and Ear Hospital, University of Melbourne, East \\ Melbourne, Australia \\ ${ }^{2}$ Department of Otorhinolaryngology, Faculty of Medicine, Khon Kaen University, Khon Kaen, Thailand \\ Email:patorn@gmail.com
}

Received 26 March 2014; revised 20 April 2014; accepted 8 May 2014

Copyright (C) 2014 by author and Scientific Research Publishing Inc.

This work is licensed under the Creative Commons Attribution International License (CC BY). http://creativecommons.org/licenses/by/4.0/

(c) (i) Open Access

\begin{abstract}
Virtual reality has been introduced to the medical field and it is now used in medical education as an alternative high fidelity simulator. The major components to successful learning that has contributed to the efficacy of the virtual reality system are the ability to provide repetitive practice under controlled environment, self-directed learning and proved construct validity. This review evaluated the validity and the efficacy of current virtual reality systems in ear, nose and throat surgery.
\end{abstract}

Keywords

Virtual Reality, Otolaryngology, Surgery

\section{Introduction}

Virtual reality simulation uses computer-generated imagery to present a training environment. It aims to increase educational outcomes over traditional methodologies [1], to enable surgical education to occur before entering theatre, and to provide a more flexible way to teach adult learners. Several potential roles have been identified for simulation in medical or surgical education, which include: 1) improved educational outcomes over traditional methodologies [1]; 2) enabling surgical education to occur before entering the operating theatre; and 3) more flexible ways to teach adult learners. Kolb [2] has suggested that knowledge is created through experience. By simulating scenarios for students, experience can be imparted to facilitate learning [3]. Simulations allow scenarios to be conducted in an arena where failure is allowed, and students can learn from these mistakes without causing harm to the actual patients [4]. This capacity to learn surgical procedures requires actual experience and a period of reflection to consolidate the students' understanding [5], and an accumulation of surgical 
experience provides an increase in performance and confidence regardless if it is simulated or real [6].

In current ear, nose and throat residency surgical training programs, residents learn by observing their mentors and practicing under their supervision. For the safety of the patients, the mentors have to ensure that residents have the capability to practice on patients prior to the surgery. Many ear, nose and throat simulator training courses were developed to facilitate the learning process for the residents. These courses range from low fidelity (e.g. rubber model for suture practice) to high fidelity (e.g. temporal bone dissection).

Many studies have found that virtual reality simulators improved trainees' operative performance. The major components to successful learning that has contributed to the efficacy of the virtual reality system are the ability to provide repetitive practice under controlled environment, self-directed learning and proved construct validity. In the early stages of learning, the simulator can be used to augment conventional training methods such as anatomical understanding, disease pathology and treatment. For the later stages of learning that require the use of clinical skills, simulators are proved to be better than conventional training techniques [7] [8]. Virtual reality simulation has been shown to improve cadaveric temporal bone dissection compared to traditional teaching methods [7] [9]. The capacity of virtual reality simulators to provide self-directed learning allows teaching to occur outside and alongside operative hours, making learning more flexible for both trainers and trainees [10] [11].

The purpose of this review was to evaluate the validity and the efficacy of current virtual reality systems in ear, nose and throat surgery.

\section{Virtual Reality in Ear Surgery}

\subsection{Temporal Bone Dissection}

Cadaveric temporal bone dissection is the main activity for residents that is used as simulation training for temporal bone surgery. Most temporal bone surgical dissection courses provide training on the principles of temporal bone and skull base surgery for practicing surgeons. The course usually emphasizes the common procedures for ear diseases. Mastoidectomy is one of the common procedures that the residents need to familiarize themselves with and have the confidence to practice without causing harm to the patient. However, there are a few setbacks associated with cadaveric dissection training. For example, the reduction in the availability of cadavers as caused by the Alder Hey controversy, together with an increased number of residency training positions, the bones have to be discarded after drilling and cannot be reused. Another concern is that illnesses may be transmitted through contact with diseased tissues and/or bodily fluids.

Many authors have addressed these problems and proposed the use of alternative simulators that can replace or augment training for cadaveric temporal bone dissection. Some authors have developed bony models to practice temporal bone dissection surgery [12], but the majority prefers to use virtual reality systems. Beside of temporal bone dissection simulators, some authors have also developed the middle ear surgery simulator for myringotomy [13] [14] and benign paroxysmal positional vertigo treatment [15].

\subsection{Current Systems}

The VOXEL-MAN Tempo Surgery Simulator is the first commercially available temporal bone virtual reality simulator [16]. Volumetric high-resolution computed tomography images of the temporal bone are used to produce a 3-dimensional representation. The surgical site is displayed in stereoscopic mode, which the user views through shutter glasses. Vital structures are color-coded. The station houses a computer with software that is linked to a force-feedback hand stylus. This stylus serves as a virtual drill, which is activated by the foot pedal to alter the appearance of the virtual temporal bone. The drill responds with forces according to the contact situation visible on screen, allowing the user to experience changes in pressure. The computer records its location, direction in space, and a number of performance measures such as excessive force or injuries relating to vital structures. The user is able to alter the surgical orientation, drill size, type, and rotation speed.

Another commercial model of temporal bone dissection simulator is the Mediseus Surgical Simulator (CSIRO/University of Melbourne Temporal Bone Simulator). This simulator consists of a simulated operating microscope. The user interacts with a 3-D volumetric virtual rendering of a cadaver temporal bone, which is controlled by the surgeon using two haptic motorized 3-D pointing devices. These devices allow the computer to track the exact movement of the tool relative to the virtual bone model. The bone model was given color and hardness properties to provide visual and tactile cues that helped to simulate real operating conditions. The co- 
loring of the bone also changes as the bone is progressively thinned over the critical structures such as the sigmoid sinus, dura, and facial nerve. Physiological functions, such as bleeding from the sigmoid sinus and facial nerve monitoring with auditory and visual feedback were also built into the simulator [9].

Many ear surgery simulators are currently under development [17]-[20]. They mostly share common features of haptic feedback. Some of them also work with acoustic feedback (Table 1).

\subsection{Efficacy of Simulator on Skills Improvement}

The VOXEL-MAN Tempo Surgery Simulator trial [21] conducted a before-after investigation and found that some surgical skills that were evaluated based on the Objective Structured Assessment of Technical Skills (OSATS) rubric experienced a significant improvement after practices were conducted by using the VR temporal bone simulator system. The OSAT scores were improved for both tegmen task from 2.125 to $3.1(P=0.026)$ and sigmoid task from 2 to $2.75(P=0.0098)$. The time to complete the tasks also decreased from 8.37 to 5.39 minutes $(P=0.018)$ for tegmen task and from 8.99 to 8.68 minutes $(P=0.594)$ for sigmoid task.

The Mediseus Surgical Simulator did further research on the improvement in the users' skills by conducting controlled trials comparing the simulator training with the conventional textbook based training and found that the participants trained on the simulator performed significantly better than the participants trained using conventional training methods [7] [8]. The experts were invited to review the participant's task through a sequence of actions (procedural score). They found that the virtual reality group was performed better than conventional training group (Table 2).

In additional to procedural skill evaluation, the authors [7] [8] also inspected the dissection results by evaluating the end-product temporal bones. The end-product score represents the combination of anatomical knowledge, procedural skill and psychomotor behavior. They found that the virtual reality group performed better than conventional training group but there was no statistically significance difference (Table 3).

The virtual simulation for temporal bone dissection [22] [23] also conducted a controlled trial comparing the virtual reality temporal bone dissection training simulator with the temporal bone dissection training. Two studies involving 92 participants used the 35-item Welling scale to determine end-product scores for the virtual reality group and cadaveric temporal bone dissection training group. There was no statistically significance difference between virtual reality group and cadaveric temporal bone dissection training group (Table 4).

The results from these studies showed that the virtual reality temporal bone dissection training is more effective than traditional training methods and as effective as cadaveric temporal bones as assessed by summative end-product score.

Table 1. Virtual reality temporal bone dissection systems.

\begin{tabular}{|c|c|c|c|}
\hline Simulators & Characteristics & Validation & Efficacy \\
\hline VOXEL-MAN Tempo Surgery & $\begin{array}{l}\text { Stereoscopic display, haptic drill, } \\
\text { foot pedal }\end{array}$ & Yes [16] & $\begin{array}{l}\text { - Pre-post study } \\
\text { - Significantly improved OSATs score }\end{array}$ \\
\hline Mediseus surgical simulator & $\begin{array}{l}\text { Stereoscopic display, haptic drill, haptic } \\
\text { suction, auditory and visual feedback }\end{array}$ & Yes [9] & $\begin{array}{l}\text { - RCTs } \\
\text { - Significantly improved procedural score }\end{array}$ \\
\hline $\begin{array}{l}\text { Virtual simulation for temporal } \\
\text { bone dissection }\end{array}$ & Stereoscopic display, haptic feedback & Yes [19] & $\begin{array}{l}\text { - RCTs } \\
\text { - Comparable to cadaveric temporal } \\
\text { bone dissection training }\end{array}$ \\
\hline $\begin{array}{l}\text { Virtual surgical training system } \\
\text { for middle ear surgery }\end{array}$ & $\begin{array}{l}\text { Stereoscopic display, haptic feedback, } \\
\text { visual feedback }\end{array}$ & Yes [17] & - Not available \\
\hline $\begin{array}{l}\text { Virtual reality temporal bone } \\
\text { dissection simulator }\end{array}$ & Haptic feedback [18] & No & - Not available \\
\hline
\end{tabular}

Table 2. Comparing the procedural score of the Mediseus virtual reality temporal bone dissection training with conventional training.

\begin{tabular}{cccc}
\hline Studies & VR $^{\mathrm{a}}$ Mean (S.D.) & Control Mean (S.D.) & Mean difference (95\% CI) \\
\hline Zhao 2011a [7] & $3.54(0.82)$ & $2.51(0.97)$ & $1.03(0.24$ to 1.82$)$ \\
Zhao 2011b [8] & $3.56(1.79)$ & $2.97(1.72)$ & $0.59(-0.95$ to 2.13$)$ \\
\hline
\end{tabular}

a. $\mathrm{VR}=$ virtual reality. 
Table 3. Comparing end-product score of the Mediseus virtual reality temporal bone dissection training with conventional training.

\begin{tabular}{cccc}
\hline Studies & VR $^{\text {a }}$ Mean (S.D.) & Control Mean (S.D.) & Mean difference (95\% CI) \\
\hline Zhao 2011a [7] & $2.79(0.79)$ & $1.98(1.12)$ & $0.81(-0.04$ to 1.66$)$ \\
Zhao 2011b [8] & $3.48(1.87)$ & $2.64(1.46)$ & $0.84(-0.63$ to 2.31) \\
\hline
\end{tabular}

a. $\mathrm{VR}=$ virtual reality.

Table 4. Comparing the end-product score of virtual reality temporal bone dissection training with temporal bone dissection training.

\begin{tabular}{cccc}
\hline Studies & VR $^{\mathbf{a}}$ Mean (S.D.) & Control Mean (S.D.) & Mean difference (95\% CI) \\
\hline Wiet 2009 [22] & $23(8.6)$ & $17(8.5)$ & $6.00(-3.68$ to 15.68$)$ \\
Wiet 2012 [23] & $2.2(0.54)$ & $2.14(0.56)$ & $0.06(-0.21$ to 0.33$)$ \\
\hline
\end{tabular}

a. $\mathrm{VR}=$ virtual reality.

\subsection{Myringotomy}

Myringotomy is the most common pediatric surgery procedure [24], which perforates the ear drum to equilibrate the pressure in the middle ear. This procedure can be conducted with or without the insertion of a tube. As there is a steep learning curve associated with the development of technical skills for middle ear surgery, there are inherent risks that patients have to face during the skills acquisition phase. The benefits of simulation training for acquiring surgical skills have also been demonstrated with previous low-fidelity models [13].

Cadavers and physical models of the tympanic membranes in the ear are the most common methods to train otolaryngology trainees in performing this operation. Neither the cadaveric nor the physical models allow for automated metrics to quantify the progress of the trainees or provide any feedback. Tactile sensation is an important feedback mechanism in middle ear surgery, hence a three-dimensional virtual reality myringotomy simulator with haptic feedback was developed [14].

A virtual reality myringotomy simulator was developed at the Auditory Biophysics Laboratory (ABL) at the University of Western Ontario (UWO) [25]. The system includes a standard 3-D gaming visor for display and a PHANTOM Omni haptic arm (SensAble Technologies, Woburn, MA) for user input and force feedback. The visor is mounted on a stand that simulates the surgical microscope. The haptic device controls a virtual blade in the scene and provides force feedback to the trainee if they collide with the ear canal, malleus, or promontory.

From the face-validation questionnaire, the virtual reality myringotomy simulator with the first to integrate a real-time deformable tympanic membrane appears to be validated for simulation. The Delaunay cutting algorithm was found to be the most realistic algorithm representing the incision during myringotomy $(P<0.05)$ [25].

\subsection{Benign Paroxysmal Positional Vertigo}

Benign paroxysmal positional vertigo (BPPV) is the most common cause of dizziness that physicians usually encounter in out-patient department settings. BPPV is a clinical syndrome that is characterized by short bursts of severe vertigo associated with changes in head orientation. The treatments for this syndrome include canalith repositioning maneuver (Epley), the Brandt-Dar off exercise, and the liberatory maneuver of Semont.

Steiner et al. [15] scanned the human membranous labyrinth at a thickness of $20 \mu \mathrm{m}$ and selected areas were segmented using the AMIRA ${ }^{\circledR} 4.1$ program. The stereoscopic representation of the inner ear was positioned according to anatomic norms. Artificial spherical surfaces representing otoliths can be loaded in the labyrinth. The model allows a basic orientation for the novice surgeons as well as complex clinical hypothesis testing for the experts.

The current virtual reality system for BPPV demonstrated how the canalith was positioned in the larynrinth by rotating the 3-D model using the mouse. The next potential step is to incorporate the mannequin that the trainee can practice the canalith repositioning maneuver on and simultaneously demonstrate the position of the canalith in the labyrinth on screen. 


\subsection{Cochlear Implantation}

Cochlear implantation inserts a cochlear prosthetic implant into the cochlea to stimulate the cochlear nerve for a patient experiencing a severe hearing impairment for mimicking the natural hearing function of the inner ear. The procedure includes a temporal bone drilling (mastoidectomy) to access the middle ear through the facial recess. The round window was identified before cochleostomy was done to access the basal turn of the cochlear. The surgeon then inserted the cochlear implant at full insertion depth. Connection tissue was placed around the cochleostomy site to seal it.

During the cochlear implant insertion, the implant disappears out of the surgeon's view soon after the initial advancement past the cochleostomy site, around the region of the basal turn. The surgeon then relies on a sense of touch for the rest of the insertion. Excess pressure after the first point of resistance may lead to induced trauma to the cochlear partition. Force feedback is required during this intricate process to minimize the risk of avoidable damage to the cochlea. Force rendering is therefore vital to accurately relay information associated with device/structure interactions for this surgical procedure.

Todd et al. [26] created a prototype simulator for prosthetic insertion. The simulator provides the ear, nose and throat surgeon with real-time visual and haptic responses during virtual cochlear implantation into a 3D model of the human scala tympani. The simulator provides the user with real-time, quantitative insertion force information and associated electrode positions as the user inserts the virtual implant into the model.

\section{Virtual Reality in Nose Surgery}

\subsection{Functional Endoscopic Sinus Surgery}

While endoscopic sinus surgery is quickly becoming popular, external approaches for nasal surgery is reserved for more complicated cases. The endoscopic approach was introduced into the rhinology field not more than 30 years ago. The efficacy of this approach is similar to the external approach but is more desirable cosmetically. Endoscopic sinus surgery training workshops are very popular among rhinologists today. The workshop usually consists of a full day of lectures and a hands-on practice to acquire the skills for endoscopic sinus surgery [27]. Functional endoscopic sinus surgery (FESS) procedure is conducted by inserting the endoscopes through the nostrils to illuminate the inside of the nasal cavity and the sinuses. The instruments can be inserted through the same nostril using another hand to manipulate the structure to get rid of diseased mucosa and maintain normal sinus drainage.

Endoscopic sinus surgery simulator was the majority in this field. Some authors also developed intranasal models and nasal packing simulators for treating epistaxis. The endoscopic sinus surgery system comprised an endoscope for the left hand and a tool manipulator for the right hand. The participants performed the surgery on the nasal model or mannequin. The endoscopic sinus surgery system has been shown to improve the performance of the participants when conducting real surgeries in operating theatre [28] [29]. However, most of the models that are used are prototypes.

\subsection{Current Systems}

The Lapsim ${ }^{\circledR}$ virtual reality simulator uses the Virtual Laparoscopic Interface non-haptic-enhanced hardware platform (Immersion Inc., San Jose, CA), which includes two endoscopic handles. The interface includes a 2600 MHz hyper-threading processor Pentium IV computer running Windows XP and is equipped with a 256 MB RAM GeForce graphic card and an 18-inch TFT monitor. The system used the Lapsim Basic Skills 3.0 software (Surgical Science Ltd., Goteburg, Sweden).

The endoscopic sinus surgical simulator (ES3) was developed by Lockheed Martin, Inc. (Akron, Ohio) with a team from the University of Washington Human Interface Technology Laboratory (Seattle), the Ohio Supercomputer Center (Columbus), and Immersion Corp (San Jose, CA). The hardware comprises 4 principal components: 1) an SGI Octane workstation (Silicon Graphics Inc., Mountain View, CA), which serves as the simulation host platform; 2) a personal computer-based haptic controller, providing control and coordination between a universal physical instrument handle and a set of virtual surgical instruments; 3) a personal computer-based voice recognition-enabled instructor, which operates the simulator by responding to spoken commands; 4) an electromechanical platform, which serves as the human interaction interface, with a replica of an endoscope, a surgical tool handle, and a rubber-headed mannequin. 
The VOXEL-MAN Sinus Surgery Simulator was adopted from their previously developed VOXEL-MAN Tempo Surgery Simulator for temporal bone surgery [30]. A 3-D model was created based on a high-resolution computed tomography. The model may be manipulated with virtual surgical tools controlled with a low-cost haptic device, which is also used to adjust microscope or endoscopic views. Visualization, haptic rendering, and tissue removal are represented with sub-voxel resolution (Table 5).

\subsection{Efficacy of Simulator on Skills Improvement}

For the physical coordination providing precise movement (psychomotor) skill, Edmond et al. assessed the efficacy of the ES3 for improving the operating room performance by evaluating the following sub-skills: navigation, injection, uncinectomy, anterior ethmoidectomy, maxillary antrostomy, orientation of image, image-task alignment, proper depth of image, tool manipulation, tool selection, tool-tool dexterity, tissue respect, surgical confidence, and case difficulty using 10 point scales [31]. Fried et al. evaluated surgical confidence and instrument manipulation performance in the operating room using scales from 1 to 10 [28]. The results showed a statistically significant difference improvement in psychomotor score for ES3 virtual reality training group in the recent study (Table 6).

For procedural skills, Fried et al. reported procedural scores using the time taken to complete the task [28]. There was a statistically significant difference in the time taken to complete task (MD 5.50 minutes, 95\% CI 1.03 to 9.97$)$.

For knowledge of specific anatomical structures, Solyar et al. [32] showed that the ES3 virtual endoscopy group had a significantly higher anatomical identification score (MD 4.3, 95\% CI 2.31 to 6.29).

\section{Virtual Reality in Throat Surgery}

Throat examination is the first step in diagnostic and treatment of the diseases. Ruthenbeck et al. [33] developed a virtual reality throat examination simulator that was capable of representing a range of throat pathologies. The computer interface employs haptic feedback to allow students to interact naturally with the software.

For the otolaryngologist, phonosurgery is one of the main procedures in this field. Surgeons require precise movements to maneuver around vocal cords under microscopy. To prevent any complications, students can first be trained using the high-fidelity vocal cord model simulator. The vocal cord in the simulator is usually made from animal tissues or synthetic materials. One study developed a complete model for laryngeal surgery. The system comprised microscopes and micro-instruments for laryngeal surgery. The results found that the physicians who were training with this simulator performed significantly better in the operating room [34].

Cricothyroidotomy is an emergency procedure that is performed when the patient's airway is blocked and less invasive attempts to clear it have failed. Cricothyroidotomy has been identified as an essential skill for military readiness. This training is relevant to more than 40,000 U.S. military medics and thousands of civilian health care providers. Current training methods use animals, cadavers and plastic mannequins. Liu et al. [35] developed

Table 5. Virtual reality endoscopic sinus surgery systems.

\begin{tabular}{|c|c|c|c|}
\hline Simulators & Characteristics & Validation & Efficacy \\
\hline ES3 & $\begin{array}{l}\text { Stereoscopic display, haptic devices, voice } \\
\text { recognition }\end{array}$ & Yes [29] & $\begin{array}{l}\text { - RCTs } \\
\text { - Significantly improved psychomotor, procedural and } \\
\text { anatomical score }\end{array}$ \\
\hline $\begin{array}{l}\text { VOXEL-MAN } \\
\text { Sinus Surgery }\end{array}$ & Stereoscopic display, haptic devices & Yes [30] & - Not available \\
\hline Lapsim & Stereoscopic display, haptic devices & Yes [36] & - Not available \\
\hline
\end{tabular}

Table 6. Comparing the psychomotor scores of ES3 virtual reality endoscopic sinus surgery training with conventional training.

\begin{tabular}{cccc}
\hline Studies & VR Mean (S.D.) & Control Mean (S.D.) & Mean difference (95\% CI) \\
\hline Edmond $2002[32]$ & $6.68(1.09)$ & $6.68(0.07)$ & $0.00(-1.51$ to 1.51$)$ \\
Fried $2010[28]$ & $6.65(2.53)$ & $2.73(1.89)$ & $3.92(2.16$ to 5.68$)$ \\
\hline
\end{tabular}


the prototype of the virtual cricothyroidotomy simulator to address the problem from other simulators e.g. animal models do not have the correct anatomy and cadavers do not have the correct physiology. Mannequins do not adequately cover the full range of anatomical variations.

Another field in laryngology is bronchoscopic surgery. The simulators in this field ranged from mannequin to virtual models. One study also developed an online virtual bronchoscopy simulator [37]. A commercial model of fiber optic bronchoscopy was the Endoscopy VR Simulator by CAE Healthcare, USA. The simulator consists of an anatomic model of the face, with an opening in one nare for nasal insertion of the fiber optic bronchoscope. The anatomic model is attached to a long compartment that houses the motion sensor system. As the user inserts and manipulates the bronchoscope, the simulator senses the movements and adjusts the screen images in real time. The simulation begins with a brief review of teaching objectives, which is followed by a patient history and relevant history and physical exam results. If X-rays or CAT scans were performed, those images are available for review. The trainee is then required to perform the selected procedure. Feedback is given during the procedure (such as an audible patient cough if topical anesthetic is insufficient) as well as after the procedure is complete. Some of the feedback is related to patient care (including how much lidocaine was administered and how many lesions were found and captured on video) [38].

\section{Conclusion}

Virtual reality surgical training in ear, nose and throat surgery is more effective than conventional training methods and could supplement standard surgical apprenticeship training to improve their technical skills before entering operating room.

\section{References}

[1] Rafiq, A., Tamariz, F., Boanca, C., Lavrentyev, V. and Merrell, R.C. (2008) Objective Assessment of Training Surgical Skills Using Simulated Tissue Interface with Real-Time Feedback. Journal of Surgical Education, 65, 270-274. http://dx.doi.org/10.1016/j.jsurg.2008.05.012

[2] Kolb, D.A. (1984) Experiential Learning: Experience as the Source of Learning and Development. Prentice-Hall, Englewood Cliffs.

[3] George, A.P. and De, R. (2010) Review of Temporal Bone Dissection Teaching: How It Was, Is and Will Be. The Journal of Laryngology \& Otology, 124, 119-125. http://dx.doi.org/10.1017/S0022215109991617

[4] Satava, R.M. (2008) Historical Review of Surgical Simulation-A Personal Perspective. World Journal of Surgery, 32, 141-148. http://dx.doi.org/10.1007/s00268-007-9374-y

[5] Rourke, L., Schmidt, M. and Garga, N. (2010) Theory-Based Research of High Fidelity Simulation Use in Nursing Education: A Review of the Literature. International Journal of Nursing Education Scholarship, 7, Article 11.

[6] Morgan, P.J., Cleave-Hogg, D., McIlroy, J. and Devitt, J.H. (2002) Simulation Technology: A Comparison of Experiential and Visual Learning for Undergraduate Medical Students. Anesthesiology, 96, 10-16. http://dx.doi.org/10.1097/00000542-200201000-00008

[7] Zhao, Y.C., Kennedy, G., Yukawa, K., Pyman, B. and O’Leary, S. (2011) Improving Temporal Bone Dissection Using Self-Directed Virtual Reality Simulation: Results of a Randomized Blinded Control Trial. Otolaryngology Head Neck Surgery, 144, 357-364. http://dx.doi.org/10.1177/0194599810391624

[8] Zhao, Y.C., Kennedy, G., Yukawa, K., Pyman, B. and O’Leary, S. (2011) Can Virtual Reality Simulator be Used as a Training Aid to Improve Cadaver Temporal Bone Dissection? Results of a Randomized Blinded Control Trial. Laryngoscope, 121, 831-837. http://dx.doi.org/10.1002/lary.21287

[9] Zhao, Y.C., Kennedy, G., Hall, R. and O’Leary, S. (2010) Differentiating Levels of Surgical Experience on a Virtual Reality Temporal Bone Simulator. Otolaryngology_Head and Neck Surgery, 143, S30-S35. http://dx.doi.org/10.1016/j.otohns.2010.03.008

[10] Sedlack, R.E., Kolars, J.C. and Alexander, J.A. (2004) Computer Simulation Training Enhances Patient Comfort during Endoscopy. Clinical Gastroenterology and Hepatology: The Official Clinical Practice Journal of the American Gastroenterological Association, 2, 348-352.

[11] Sedlack, R.E. and Kolars, J.C. (2004) Computer Simulator Training Enhances the Competency of Gastroenterology Fellows at Colonoscopy: Results of a Pilot Study. The American Journal of Gastroenterology, 99, 33-37. http://dx.doi.org/10.1111/j.1572-0241.2004.04007.x

[12] Varadarajan, V., Verma, R. and Auccott, W. (2010) The Portable Temporal Bone Lab-A Useful Training Adjunct for the ENT Trainee. Clinical Otolaryngology, 35, 449-450. http://dx.doi.org/10.1111/j.1749-4486.2010.02203.x 
[13] Sowerby, L.J., Rehal, G., Husein, M., Doyle, P.C., Agrawal, S. and Ladak, H.M. (2010) Development and Face Validity Testing of a Three-Dimensional Myringotomy Simulator with Haptic Feedback. Journal of Otolaryngology-Head \& Neck Surgery, 39, 122-129.

[14] Wheeler, B., Doyle, P.C., Chandarana, S., Agrawal, S., Husein, M. and Ladak, H.M. (2010) Interactive Computer-Based Simulator for Training in Blade Navigation and Targeting in Myringotomy. Computer Methods and Programs in Biomedicine, 98, 130-139. http://dx.doi.org/10.1016/j.cmpb.2009.09.010

[15] Steiner, K.V., Teixido, M., Kung, B., Sorensen, M., Forstrom, R. and Coller, P. (2007) A Virtual-Reality Approach for the Treatment of Benign Paroxysmal Positional Vertigo. Studies in Health Technology and Informatics, 125, 451-453.

[16] Arora, A., Khemani, S., Tolley, N., Singh, A., Budge, J., Varela, D.A., Francis, H.W., Darzi, A. and Bhatti, N.I. (2012) Face and Content Validation of a Virtual Reality Temporal Bone Simulator. Otolaryngology—Head and Neck Surgery, 146, 497-503. http://dx.doi.org/10.1177/0194599811427385

[17] Agus, M., Giachetti, A., Gobbetti, E., Zanetti, G., Zorcolo, A., Picasso, B. and Franceschini, S.S. (2003) A Haptic Model of a Bone-Cutting Burr. Studies in Health Technology and Informatics, 94, 4-10.

[18] Kuppersmith, R.B., Johnston, R., Moreau, D., Loftin, R.B. and Jenkins, H. (1997) Building a Virtual Reality Temporal Bone Dissection Simulator. Studies in Health Technology and Informatics, 39, 180-186.

[19] Stredney, D., Wiet, G.J., Bryan, J., Sessanna, D., Murakami, J., Schmalbrock, P., Powell, K. and Welling, B. (2002) Temporal Bone Dissection Simulation-An Update. Studies in Health Technology and Informatics, 85, 507-513.

[20] Agus, M., Giachetti, A., Gobbetti, E., Zanetti, G., Zorcolo, A., John, N.W. and Stone, R.J. (2002) Mastoidectomy Simulation with Combined Visual and Haptic Feedback. Studies in Health Technology and Informatics, 85, 17-23.

[21] Francis, H.W., Malik, M.U., Varela, D.A.D.V., Barffour, M.A., Chien, W.W., Carey, J.P., Niparko, J.K. and Bhatti, N.I. (2012) Technical Skills Improve after Practice on Virtual-Reality Temporal Bone Simulator. The Laryngoscope, 122, 1385-1391. http://dx.doi.org/10.1002/lary.22378

[22] Wiet, G.J., Rastatter, J.C., Bapna, S., Packer, M., Stredney, D. and Welling, D.B. (2009) Training Otologic Surgical Skills through Simulation-Moving toward Validation: A Pilot Study and Lessons Learned. Journal of Graduate Medical Education, 1, 61-66. http://dx.doi.org/10.4300/01.01.0010

[23] Wiet, G.J., Stredney, D., Kerwin, T., Hittle, B., Fernandez, S.A., Abdel-Rasoul, M. and Welling, D.B. (2012) Virtual Temporal Bone Dissection System: OSU Virtual Temporal Bone System: Development and Testing. The Laryngoscope, 122, S1-S12. http://dx.doi.org/10.1002/lary.22499

[24] McIsaac, W.J., Coyte, P.C., Croxford, R., Asche, C.V., Friedberg, J. and Feldman, W. (2000) Otolaryngologists’ Perceptions of the Indications for Tympanostomy Tube Insertion in Children. Canadian Medical Association or Its Licensor, 162, 1285-1288.

[25] Ho, A.K., Alsaffar, H., Doyle, P.C., Ladak, H.M. and Agrawal, S.K. (2012) Virtual Reality Myringotomy Simulation with Real-Time Deformation: Development and Validity Testing. The Laryngoscope, 122, 1844-1851. http://dx.doi.org/10.1002/lary.23361

[26] Todd, C. (2011) Real-Time Haptic Modeling and Simulation for Prosthetic Insertion. World Academy of Science, Engineering and Technology, 49, 343-351.

[27] Arora, H., Uribe, J., Ralph, W., Zeltsan, M., Cuellar, H., Gallagher, A. and Fried, M.P. (2005) Assessment of Construct Validity of the Endoscopic Sinus Surgery Simulator. Archives of Otolaryngology_Head and Neck Surgery, 131, 217221. http://dx.doi.org/10.1001/archotol.131.3.217

[28] Fried, M.P., Sadoughi, B., Gibber, M.J., Jacobs, J.B., Lebowitz, R.A., Ross, D.A., Bent 3rd, J.P., Parikh, S.R., Sasaki, C.T. and Schaefer, S.D. (2010) From Virtual Reality to the Operating Room: The Endoscopic Sinus Surgery Simulator Experiment. Otolaryngology—Head and Neck Surgery, 142, 202-207. http://dx.doi.org/10.1016/j.otohns.2009.11.023

[29] Fried, M.P., Sadoughi, B., Weghorst, S.J., Zeltsan, M., Cuellar, H., Uribe, J.I., Sasaki, C.T., Ross, D.A., Jacobs, J.B., Lebowitz, R.A., et al. (2007) Construct Validity of the Endoscopic Sinus Surgery Simulator: II. Assessment of Discriminant Validity and Expert Benchmarking. Archives of Otolaryngology—Head \& Neck Surgery, 133, 350-357. http://dx.doi.org/10.1001/archotol.133.4.350

[30] Tolsdorff, B., Pommert, A., Hohne, K.H., Petersik, A., Pflesser, B., Tiede, U. and Leuwer, R. (2010) Virtual Reality: A New Paranasal Sinus Surgery Simulator. The Laryngoscope, 120, 420-426.

[31] Edmond Jr., C.V. (2002) Impact of the Endoscopic Sinus Surgical Simulator on Operating Room Performance. The Laryngoscope, 112, 1148-1158. http://dx.doi.org/10.1097/00005537-200207000-00002

[32] Solyar, A., Cuellar, H., Sadoughi, B., Olson, T.R. and Fried, M.P. (2008) Endoscopic Sinus Surgery Simulator as a Teaching Tool for Anatomy Education. The American Journal of Surgery, 196, 120-124. http://dx.doi.org/10.1016/j.amjsurg.2007.06.026

[33] Ruthenbeck, G.S., Owen, H. and Reynolds, K.J. (2008) A Virtual Reality Throat Examination Simulation. Studies in Health Technology and Informatics, 132, 433-435. 
[34] Contag, S.P., Klein, A.M., Blount, A.C. and Johns III, M.M. (2009) Validation of a Laryngeal Dissection Module for Phonomicrosurgical Training. The Laryngoscope, 119, 211-215. http://dx.doi.org/10.1002/lary.20018

[35] Liu, A., Bhasin, Y. and Bowyer, M. (2005) A Haptic-Enabled Simulator for Cricothyroidotomy. Studies in Health Technology and Informatics, 111, 308-313.

[36] van Dongen, K.W., Ahlberg, G., Bonavina, L., Carter, F.J., Grantcharov, T.P., Hyltander, A., Schijven, M.P., Stefani, A., van der Zee, D.C. and Broeders, I.A. (2011) European Consensus on a Competency-Based Virtual Reality Training Program for Basic Endoscopic Surgical Psychomotor Skills. Surgical Endoscopy, 25, 166-171. http://dx.doi.org/10.1007/s00464-010-1151-6

[37] Duffy, C. and Myles, P.S. (2008) Review: Thoracic-Anesthesia.com. Journal of Cardiothoracic and Vascular Anesthesia, 22, 644. http://dx.doi.org/10.1053/j.jvca.2007.09.005

[38] Dalal, P.G., Dalal, G.B., Pott, L., Bezinover, D., Prozesky, J. and Murray, W.B. (2011) Learning Curves of Novice Anesthesiology Residents Performing Simulated Fibreoptic Upper Airway Endoscopy. Canadian Journal of Anesthesia/Journal Canadien d'Anesthésie, 58, 802-809. http://dx.doi.org/10.1007/s12630-011-9542-2 